\title{
ABCA1 mRNA and Protein Distribution Patterns Predict Multiple Different Roles and Levels of Regulation
}

\author{
Cheryl L. Wellington, Elizabeth K. Y. Walker, Agripina Suarez, Anita Kwok, \\ Nagat Bissada, Roshni Singaraja, Yu-Zhou Yang, Lin-Hua Zhang, Erick James, \\ Janet E. Wilson, Omar Francone, Bruce M. McManus, and Michael R. Hayden
}

Centre for Molecular Medicine and Therapeutics (CLW, AK, NB, RS, Y-ZY, EJ, MRH), University of British Columbia McDonald Research Laboratories/The iCAPTURE Centre, Department of Pathology and Laboratory Medicine (EKYW, AS, JEW, BMM), St. Paul's Hospital/Providence Health Care-University of British Columbia, Xenon Genetics, Inc. (L-HZ, MRH), Vancouver, British Columbia, Canada; and Pfizer Global Research and Development (OF), Groton, Connecticut

\begin{abstract}
SUMMARY: Mutations in ABCA1 cause the allelic disorders familial hypolipoproteinemia and Tangier Disease. To identify where ABCA1 was likely to have a functional role, we determined the cellular and tissue-specific patterns of murine ABCA1 expression. RT-PCR and Western blot analysis on dissected murine tissues demonstrated broad expression of ABCA1 mRNA and protein in many tissues with prominent protein expression in liver, testis, and adrenal tissue. In situ hybridization and immunohistochemistry experiments demonstrated specific patterns of ABCA1 expression at the cellular level, with hepatocytes, the epithelial lining of the digestive system and bladder, the proximal convoluted tubule of the kidney, and Purkinje and cortical pyramidal neurons containing abundant ABCA1 protein. Significant discordance between relative mRNA and protein expression patterns suggests the possibility of post-transcriptional regulation of ABCA1 expression in selected cells or tissues. We also show that ABCA1 protein levels are up-regulated specifically in the liver after exposure to an atherogenic diet for 7 days, supporting a major role for the liver in dietary modulation of HDL-C levels. Our observations show that ABCA1 is expressed in a pattern consistent with its role in HDL-C metabolism. Additionally, ABCA1 may have important functional roles in other cell types independent of HDL-C regulation. (Lab Invest 2002, 82:273-283).
\end{abstract}

\begin{abstract}
$A$ BCA1 is an ATP-binding cassette transporter that is crucial for the production of high-density lipoproteins (HDL) (Broccardo et al, 1999; Klein et al, 1999; Oram and Vaughan, 2000; Schmitz et al, 2000). ABCA1 acts to transfer phospholipid and cholesterol onto apo-A1 during reverse cholesterol transport. Defects in ABCA1 result in the allelic disorders familial hypoalphalipoproteinemia (FHA) or the more severe disorder Tangier Disease (TD), that are characterized by greatly reduced level of HDL-C cholesterol in plasma, impaired cholesterol efflux, and a tendency to accumulate intracellular cholesterol ester (Bodzioch et al, 1999; Brooks-Wilson et al, 1999; Marcil et al, 1999; Rust et al, 1999). Because high levels of HDL-C are atheroprotective, there is considerable interest in developing compounds that act to increase ABCA1 expression and thereby raise plasma HDL-C levels. Identification of the expression patterns of ABCA1 is
\end{abstract}

Received September 27, 2001.

This work was supported by the Canadian Institutes of Health Research (no. $20 R 91253$ to MRH), the Heart and Stroke Foundation of Canada (no. $20 R 53911$ to BMM and MRH), the Canadian Networks of Centres of Excellence (NCE Genetics, MRH), and Xenon Genetics, Inc. (BMM, MRH). MRH is an established investigator of the BC Children's Hospital and is a holder of a Canadian Institutes of Health Research Chair. Address reprint requests to: Dr. Michael R. Hayden, 950 West 28th Avenue,Vancouver, BC, CanadaV5Z 4H4.E-mail:mrb@cmmt.ubc.ca an important indicator of sites of action of ABCA1 and a prerequisite for safe and effective use of such potential therapeutic approaches.

Previous reports have suggested that ABCA1 is expressed ubiquitously, although most of these studies have been performed using qualitative approaches and base their conclusions solely on mRNA expression data (Kaminski et al, 2000; Langmann et al, 1999). In these studies, the use of nonnumeric scales does not permit the relative level of mRNA to be compared among different tissues. More importantly, however, mRNA is not a uniformly good predictor of protein levels (Gygi et al, 1999). For example, expression of mRNA can vary 20 -fold among comparably expressed proteins, and protein expression can vary 30 -fold among comparably expressed mRNAs (Gygi et al, 1999). Therefore, it is essential to determine both mRNA and protein distribution patterns to characterize tissue-specific regulatory pathways. Furthermore, it is important to examine mRNA and protein expression patterns using histological approaches to identify particular cell types within a given tissue that may be enriched in ABCA1.

To this end, we provide the first identification of global ABCA1 protein and mRNA expression patterns in the mouse, using RT-PCR and Western blotting of whole murine tissues as well as in situ hybridization (ISH) and immunohistochemistry (IHC). We show that 
murine $A B C A 1$ is expressed in tissues known to be involved in regulation of $\mathrm{HDL}-\mathrm{C}$ homeostasis as well as in tissues not involved in reverse cholesterol transport, suggesting that ABCA1 is likely to have functional roles in addition to cholesterol/phospholipid efflux. In many tissues, the presence of ABCA1 mRNA coincides with $A B C A 1$ protein. However, in certain cells and tissues there is notable discordance between $A B C A 1$ mRNA and protein expression, suggesting that regulation of $A B C A 1$ expression may occur at both transcriptional and post-transcriptional levels. Furthermore, exposure of mice to an atherogenic diet for 7 days resulted in a 3.2-fold upregulation of $A B C A 1$ protein expression most obviously in the liver, supporting the existence of tissuespecific regulatory pathways involved in ABCA1 expression.

\section{Results}

\section{Tissue Distribution of Murine ABCA1 mRNA}

The global distribution pattern of adult murine ABCA1 mRNA expression was generated using RT-PCR to compare ABCA1 transcript levels relative to $18 \mathrm{~s} r R N A$ levels in tissues. The intensity of the ABCA1 product was normalized relative to that of the $18 \mathrm{~s}$ rRNA product by densitometric imaging, and the relative amount of ABCA1:18s RNA was compared across many tissues obtained from four individual animals maintained on a chow diet. The relative abundance of ABCA1 mRNA in murine tissues was divided into three groups (Fig. 1). Highest ABCA1 mRNA expression was observed in liver, kidney, adrenal, heart, bladder, testis, and brain. Moderate expression was observed in lung, adipose, esophagus, stomach, and small intestine. Large intestine, skeletal muscle, thymus,

\section{Tissue distribution of ABCA1 mRNA}

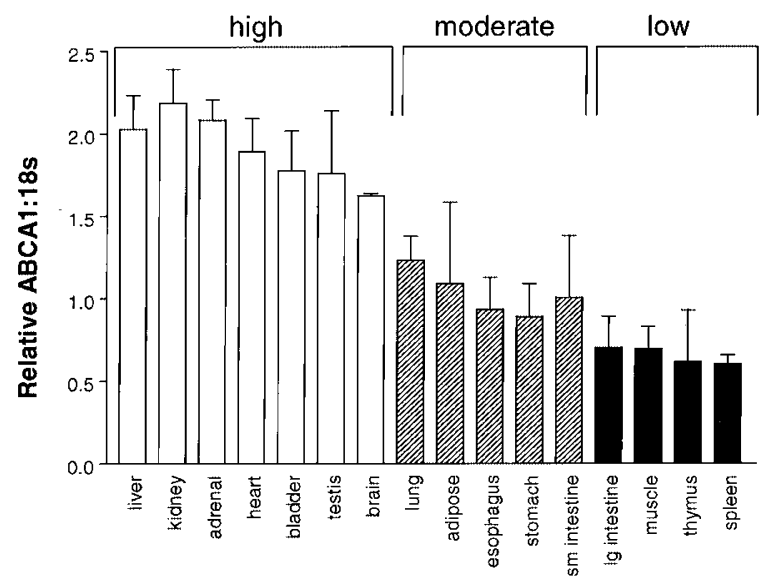

Figure 1.

Tissue distribution of murine ABCA1 mRNA. Tissues were obtained from four individual male mice maintained on a chow diet. Bars represent the mean of at least four independent determinations of the ratio of ABCA1 mRNA to 18s rRNA (using the maximal concentration of 18s rRNA competimer) as determined by RT-PCR and then by densitometric quantitation of band intensity on an ethidium bromide-stained agarose gel. and spleen consistently contained relatively low levels of ABCA1 mRNA.

These experiments showed that ABCA1 mRNA is expressed widely throughout the body but at different levels in different tissues. However, because expression of mRNA does not necessarily predict protein expression (Gygi et al, 1999), we then determined the tissue-specific expression patterns of ABCA1 protein using two $\mathrm{ABCA} 1$-specific $\mathrm{C}$-terminal antibodies.

\section{Validation of ABCA1 Antibodies}

Because ABCA1 is one of approximately 100 members of the ATP-binding cassette superfamily of proteins, multiple approaches were used to verify specific immunoreactivity toward ABCA1 from immunoreactivity to other related proteins of very similar size. In one approach, ABCA1 was radiolabeled with $S^{35}$ during in vitro translation from human cDNA expression constructs comprising N-terminal, C-terminal, or fulllength ABCA1. Before Western analysis, membranes were exposed to film to detect the radiolabeled protein (Fig. 2A, left panel). Then, a Western blot was performed using the test antisera and a second film was generated to detect protein(s) recognized by the antisera (Fig. 2A, right panel). Overlaying the two films allowed verification of specific immunoreactivity present in the antisera that corresponded to the presence of C-terminal or full-length ABCA1 synthesized in vitro. The reticulocyte lysate was also found to contain a 90-100 kD cross-reactive species detected by the crude polyclonal antisera.

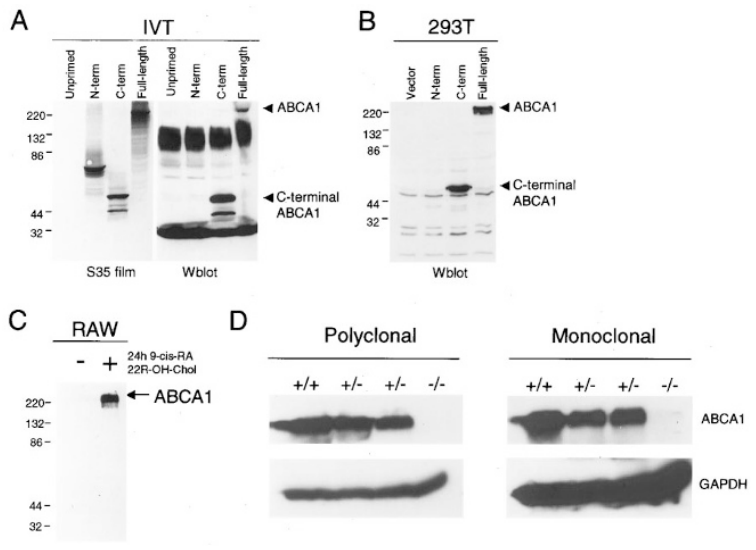

\section{Figure 2.}

Validation of ABCA1 antibodies. A, $S^{35}$-labeled protein generated by in vitro translation (IVT) of $\mathrm{N}$-terminal $(\mathrm{N}$-term), $\mathrm{C}$-terminal $(\mathrm{C}$-term), and full-length ABC1 constructs, and a no DNA control (unprimed). The left panel is an autographic image of the radiolabeled protein before Western blotting. The right panel is the same filter probed with crude polyclonal anti-ABCA1 antiserum. B, Western blot of crude anti-ABCA1 antisera on protein lysates from human embryonic kidney 293T cells transiently transfected with empty vector (Vector), N-terminal ( $\mathrm{N}$-term), C-terminal (C-term), and full-length ABCA1 expression constructs. $C$, Western blot of RAW murine macrophages with affinity-purified $A B C A 1$ antibody showing up-regulation of $A B C A 1$ after 24-hour treatment with $10 \mu \mathrm{M}$ each 9-cis-retinoic acid and 22-R-OHcholesterol. D, Whole liver lysates prepared from wild-type $(+/+)$, hemizygous (+/-), and ABCA1-null (-/-) mice, probed with affinity purified polyclonal ABCA1 antibody (left panel) or monoclonal ABCA1 antibody (right panel). 
Transient transfection of the same expression constructs into 293T cells generated truncated and fulllength proteins that were also recognized by crude antisera (Fig. 2B). Affinity purification removed crossreactive species and allowed specific detection of endogenous levels of $A B C A 1$, as demonstrated by the presence of a single band induced by 9-cis-retinoic acid, 22-R-OH-Chol in RAW murine macrophages that is detected by the affinity-purified polyclonal antibody (Fig. 2C). Finally, liver lysates prepared from wild-type or ABCA1-deficient mice were used to demonstrate detection of ABCA1 protein in wild-type or hemizygous animals and the absence of detectable signal in ABCA1-deficient animals (Fig. 2D). Taken together, these results demonstrate that these ABCA1 antibodies specifically recognize human or murine $A B C A 1$ protein without detectable cross-reactivity. Because the monoclonal antibody was shown to be approximately three times more sensitive toward an ABCA1 fusion protein by ELISA assay (data not shown), the monoclonal antibody was used for most of the experiments discussed below.

\section{Tissue Distribution of ABCA1 Protein}

Having verified their specificity, both antibodies were then used to determine the tissue distribution of ABCA1 protein obtained from at least six individual adult mice. Animals were maintained on a chow diet and each tissue was analyzed by Western blot and normalized to glyceraldehyde phosphate dehydrogenase (GAPDH) levels. The polyclonal and monoclonal antibodies gave indistinguishable results. Liver, testis, and adrenal gland contained the highest levels of ABCA1 protein, whereas lung, adipose, bladder, spleen, brain, thymus, and small intestine contained moderate amounts of ABCA1 (Fig. 3). The lowest levels of $A B C A 1$ protein were observed in large intestine, kidney, stomach, heart, esophagus, and skeletal muscle.

\section{Concordance of ABCA1 mRNA and Protein Expression in Murine Tissues}

Overall, the relative abundance of ABCA1 mRNA does not accurately predict ABCA1 protein levels $\left(r^{2}=\right.$ 0.199 ). However, there are tissues with highly concordant mRNA and protein expression including liver, testis, adrenal, lung, adipose, intestine, and skeletal muscle $\left(r^{2}=0.776\right.$; Table 1). In other tissues including kidney, heart, bladder, brain, esophagus, stomach, thymus, and spleen, the relative abundance of ABCA1 mRNA and protein are not well correlated $\left(r^{2}=0.045\right.$, Table 1). These results suggest that post-transcriptional mechanisms, such as regulation of ABCA1 translation or protein stability, may partly determine the level of ABCA1 protein expression in selected regions. However, because it is possible that $A B C A 1$ expression also varies by cell type within tissues, we then characterized the cellular expression patterns of ABCA1 by performing in situ hybridization and immunohistochemistry on wild-type FVB murine sections.

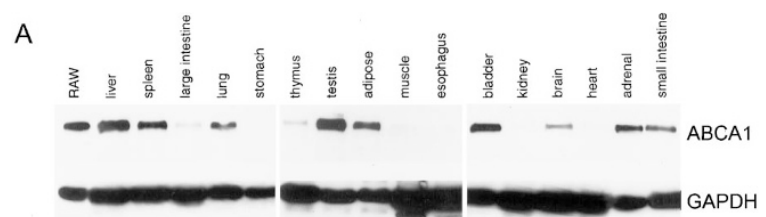

B

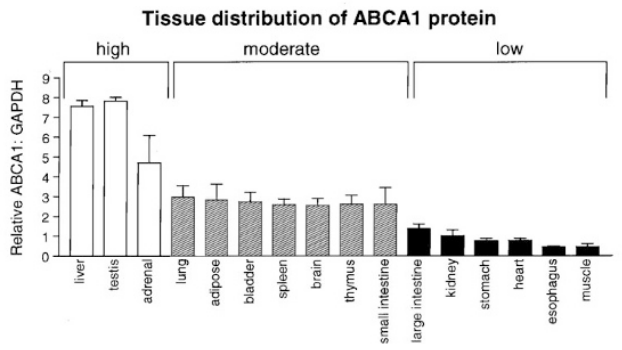

Figure 3.

Tissue distribution of murine ABCA1 protein. A, Western blot of whole-cell lysates prepared from adult murine tissues showing $A B C A 1$ protein in multiple tissues. Lysates from RAW murine macrophages in which ABCA1 expression was stimulated with 9-cis-retinoic acid, 22- $\mathrm{R}-\mathrm{OH}$-cholesterol stimulated served as a positive control. After transfer to a PVDF membrane, the gel was cut in half and the top and bottom halves probed with $A B C A 1$ and glyceraldehyde phosphate dehydrogenase (GAPDH) antibodies, respectively. B, Bars represent the mean of densitometric quantitation of $A B C A 1$ relative to GAPDH protein levels from gels obtained from at least six independent animals.

\section{Cellular Distribution of ABCA1 mRNA}

Expression of ABCA1 mRNA within individual cells from various tissues was determined by in situ hybridization using a specific $\mathrm{N}$-terminal antisense ABCA1 probe. Tissues were obtained from adult mice maintained on a chow diet and all were found to have normal morphology as determined by hematoxylineosin staining (not shown). One pattern that emerged from this analysis was that the lining epithelia of many tissues, including intestine, esophagus, stomach, and bladder, express high levels of ABCA1 mRNA (Fig. 4A and not shown). For example, ABCA1 mRNA was localized to both the lining epithelium and lamina propria of the murine small intestine (Fig. 4A). Interestingly, maximal mRNA expression was observed in the crypts of Lieberkühn and diminished toward the tips of the villi. No signal was detected using the control probe (Fig. 4B). Similar results were obtained for other gut tissues, including the large intestine and stomach (not shown). In the liver, ABCA1 mRNA was expressed in many cell types, including hepatocytes, particularly near the central lobule, and sinusoid lining cells, including F4/80-positive Kupffer cells (Fig. 4C). In the cerebellum, ABCA1 mRNA was abundant in Purkinje cell bodies, whereas little ABCA1 mRNA was detected in cerebellar granule neurons (Fig. 4D). ABCA1 mRNA was also identified in cortical pyramidal neurons and, to a much lesser extent, in glia (not shown). The tubular epithelium of the kidney was notable for expression of two different levels of ABCA1 mRNA (Fig. $4 \mathrm{E})$. Most tubules expressed moderate amounts of ABCA1 mRNA, and the epithelia of proximal convoluted tubules stained intensely positively. In contrast, ABCA1 is expressed very poorly in glomeruli, and only a few individual cells, likely rare F4/80-positive macrophages, were observed to contain ABCA1 mRNA 
Table 1. Concordance Between ABCA1 mRNA and Protein Expression (Overall $r^{2}=0.199$ )

\begin{tabular}{|c|c|c|c|c|c|}
\hline \multicolumn{2}{|c|}{ Concordant $\left(r^{2}=0.776\right)$} & \multirow[b]{2}{*}{ Protein } & \multicolumn{2}{|c|}{ Discordant $\left(r^{2}=0.045\right)$} & \multirow[b]{2}{*}{ Protein } \\
\hline Tissue & mRNA & & Tissue & mRNA & \\
\hline Liver & High & High & Kidney & High & Low \\
\hline Testis & High & High & Heart & High & Low \\
\hline Adrenal & High & High & Bladder & High & Moderate \\
\hline Lung & Moderate & Moderate & Brain & High & Moderate \\
\hline Sm intestine & Moderate & Moderate & Esophagus & Moderate & Low \\
\hline Adipose & Moderate & Moderate & Stomach & Moderate & Low \\
\hline Lg intestine & Low & Low & Thymus & Low & Moderate \\
\hline Sk muscle & Low & Low & Spleen & Low & Moderate \\
\hline
\end{tabular}

Table 2. Cellular Expression of ABCA1

\begin{tabular}{|c|c|c|}
\hline Tissue & mRNA & Protein \\
\hline Esophagus & Lining epithlium & Lining epithelium \\
\hline Stomach & Lining epithlium & Lining epithelium \\
\hline Small intestine & $\begin{array}{l}\text { Lining epithelium (particularly crypts), lamina } \\
\text { propria throughout }\end{array}$ & $\begin{array}{l}\text { Surface epithelium mostly in apical region, weak in } \\
\text { lamina propria }\end{array}$ \\
\hline Large intestine & Lining epithelium, lamina propria throughout & Surface epithelium, weak in lamina propria \\
\hline Liver & Hepatocytes, some Kupffer cells & Hepatocytes, some Kupffer cells \\
\hline Bladder & Lining epithlium & Lining epithelium \\
\hline Cerebellum & Purkinje cells & Purkinje cell bodies and arbor \\
\hline Cortex & Cortical neurons & Cortical neurons \\
\hline Adrenal & Clusters of medullary cells & Clusters of medullary cells \\
\hline Kidney & Proximal convoluted tubule & Proximal convoluted tubule \\
\hline Adipose & Interstitial fibroblasts & Interstitial fibroblasts and vessels \\
\hline Lung & Alveolar macrophages, small bronchioles & Alveolar macrophages, small bronchioles \\
\hline Spleen & $\begin{array}{l}\text { Cells within follicles, splenic macrophages, } \\
\text { red pulp }\end{array}$ & Cells within follicles, splenic macrophages, red pulp \\
\hline Cardiomyocytes & Very low expression, diffuse & Very low expression, diffuse \\
\hline Skeletal muscle & Very low expression, diffuse & Patchy in longitudinal fibres \\
\hline
\end{tabular}

(Fig. 4E). Finally, although moderate levels of ABCA1 were detected in bulk adipose tissue, ISH revealed that adipocytes themselves were devoid of ABCA1 mRNA and that only interstitial fibroblasts in connective tissue and occasional small vessels expressed ABCA1 mRNA (Fig. 4F).

Elsewhere, ABCA1 mRNA was identified in alveolar macrophages and the epithelium of small bronchioles in the lung (not shown). In the adrenal gland, medullary cells were found to express more ABCA1 mRNA than cortical cells (not shown). In the spleen, highest ABCA1 mRNA expression was observed in the follicles, with enhanced staining of splenic macrophages as well as in red pulp (not shown). ABCA1 protein in cardiomyocytes and skeletal muscle was identified in distinct patches of cells, with most signal detected along striations of longitudinally cut skeletal muscle fibres (not shown).

\section{Cell-Specific Patterns of ABCA1 Protein Expression}

Immunohistochemistry on adult murine tissues was performed to localize ABCA1 protein to specific cell types. Both the monoclonal and polyclonal ABCA1 antibodies gave comparable results. In liver, ABCA1 protein is found in most hepatocytes, although the level of $A B C A 1$ protein expression varies among hepatocytes (Fig. 5, A and D). ABCA1 protein is also found in sinusoidal lining cells that are likely Kupffer cells due to $F 4 / 80$ reactivity, but is not expressed in the lining endothelium of portal vessels (Fig. 5, A and C). Abundant expression of ABCA1 protein in the liver supports a major role for hepatocytes in the regulation of HDL-C levels. Furthermore, as shown below, $A B C A 1$ protein expression is modulated specifically in the liver in response to diet, providing further evidence for liver-specific functions of ABCA1 in lipid metabolism.

In the small intestine, ABCA1 protein is most abundant in the villus lining epithelium (Fig. 6, A and C). In contrast to ABCA1 mRNA that was predominant in the dividing cells of the crypts of Lieberkühn, ABCA1 protein within the gut epithelium was expressed throughout the length of the villus epithelium. These results suggest that although dividing cells may transiently express ABCA1 mRNA, ABCA1 protein is stable and persists throughout the lifetime of the gut epithelial cell. ABCA1 protein was also present in individual cells within the lamina propria that are likely interstitial F4/80-positive macrophages (Fig. $6 \mathrm{E})$. The underlying smooth muscle cells and basal 

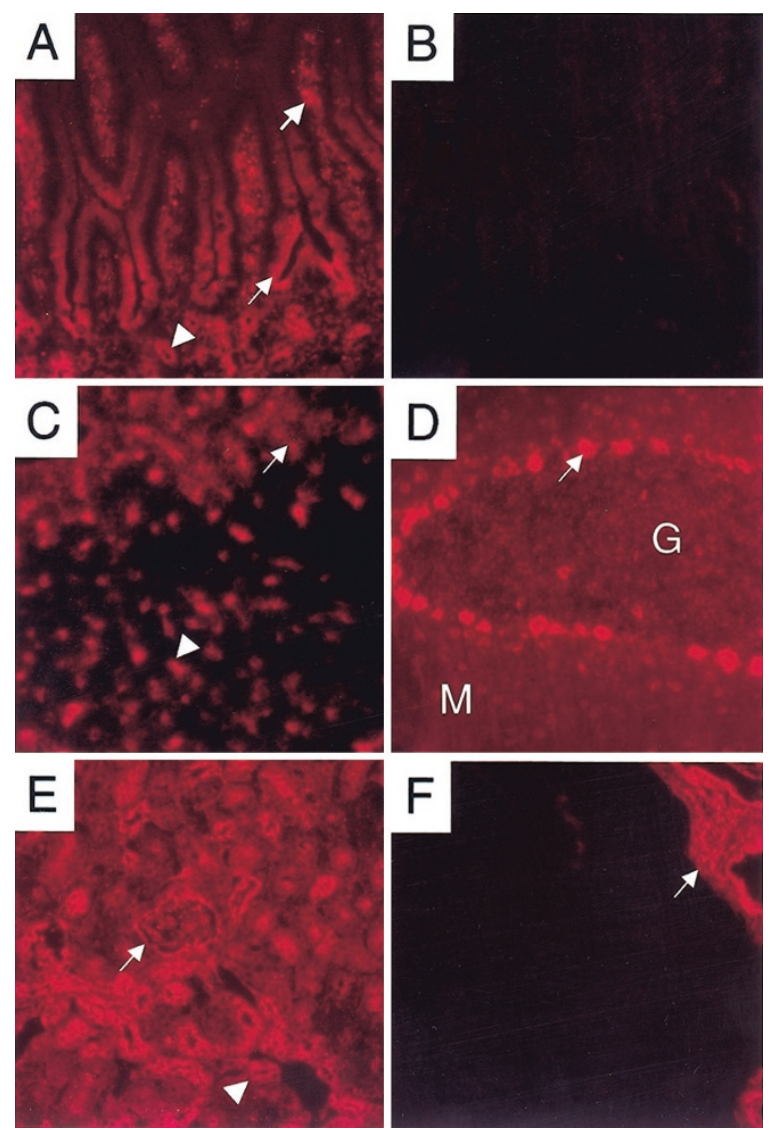

Figure 4.

In situ hybridization of various murine tissues for ABCA1 mRNA. A, Murine small intestine showing distinct fluorescent positivity in this darkfield panel. Importantly, there are apparently different locales of bright positivity. First, basal glands that are germinative for epithelium that ultimately lines the villi of the small intestine have brightly positive epithelium. In these basal glands, the crypts of Lieberkühn (arrows) deliver secretions to the intervillar spaces, and Brunner's glands (arrowhead) deliver their secretions into the crypts; both types of cells stain very positively for ABCA1 mRNA. The positivity of the epithelial cells, including the surface absorptive cells and enteroendocrine cells, diminishes in a graded fashion toward the tips of the villi. The lamina propria was uniformly labeled. B, Negative control panel showing no staining for the control riboprobe in small intestine. Similar results were observed in all other tissues examined (data not shown). C, Murine liver. The extent of positivity is most readily seen in the darkfield image with the anti-sense probe. Diffuse positivity is visible in hepatocytes (arrow). Positivity is also visible in sinusoid-lining phagocytic cells (Kupffer cells, arrowhead), later confirmed through serial sections to be positively (F4/80) staining macrophages. D, Murine cerebellum. The most striking cells with respect to positivity are the Purkinje cells (arrow) at the most superficial margin between the granular layer $(G)$ and the molecular layer $(M)$. E, Murine kidney. Moderate positivity is seen in the epithelial cells of the proximal convoluted tubules (arrowhead). Importantly, not all tubular epithelium is of a similar positivity. There is also positivity in certain interstitial small blood vessels. The glomeruli (arrow) are generally only weakly positive and only certain cells within them appear to be positive for ABCA1 mRNA. F, Murine adipose tissue. The adipocytes themselves are absent for ABCA1 mRNA. Small insterstitial blood vessels as well as overlying or adjacent connective tissue (arrow) is variably positive.

glands expressed virtually no ABCA1 protein (Fig. 6A). Similar results were obtained for other gut tissues, including the large intestine and stomach (not shown).

In the kidney, ABCA1 protein was found predominantly in the proximal convoluted tubule compared with the distal convoluted tubule (Fig. 7A). Glomeruli

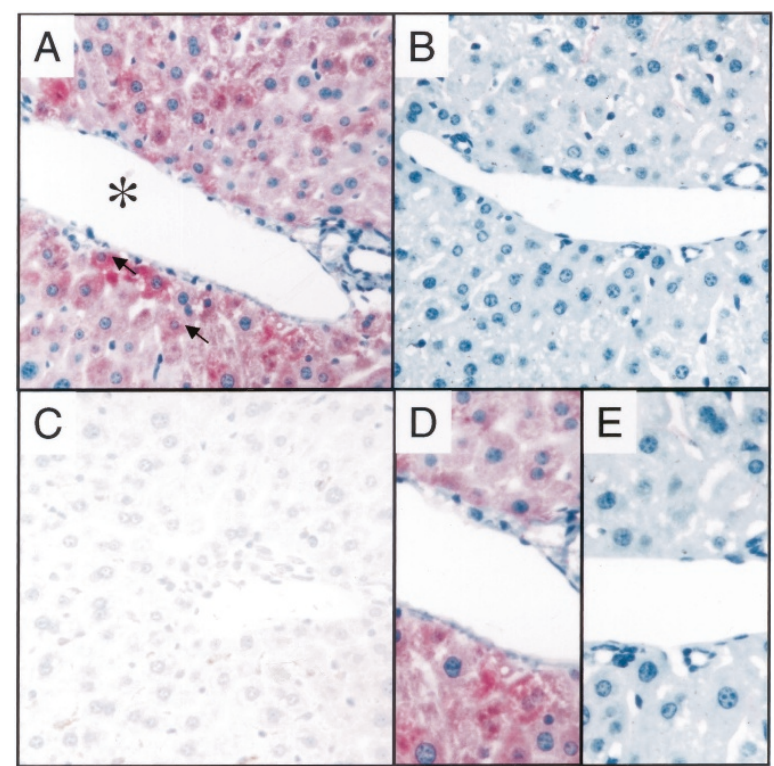

Figure 5 .

Immunoreactivity of murine liver for ABCA1 protein. A, Positivity is noted in most hepatocytes with a somewhat uneven degree of expression (arrows). The asterisk designates a small intrahepatic vein in which the lining endothelium appears to be negative for ABCA1 staining. B, The negative control for Panel A. C, Staining with an antibody to macrophages (F4/80) reveals slight positivity of sinusoidal lining cells, apparent Kupffer cells. Otherwise the liver is negative. $D$, Higher magnification of liver stained for ABCA1 protein illustrating uneven accumulation of ABCA1 protein in hepatocytes. E, The negative control for Panel D.

appeared to have little ABCA1 protein except for a few isolated cells (Fig. 7A). Collecting ducts were only faintly positive and small vessels were negative. Additionally, the thick ascending limb of the loop of Henle within the deep medulla contained abundant ABCA1 protein compared with the cortex (Fig. 7C).

In the brain, high levels of ABCA1 protein were found within Purkinje cell bodies and weaker staining extended throughout the Purkinje arbor within the molecular layer of the cerebellum (Fig. 8A). In contrast, most murine cerebellar granule neurons within the granular layer contained very little ABCA1 protein (Fig. $8 A)$. ABCA1 protein was also highly expressed in the cell bodies of large pyramidal cortical neurons (Fig. $8 \mathrm{C}$ ), and also within striatal neurons (Singaraja et al, 2001). In contrast, most glia were found to contain little or no ABCA1 protein (Fig. 8C).

In adipose tissue, most of the ABCA1 protein was found between adipose cells, likely in interstitial fibroblastic cells (Fig. 9A). In the pancreas, ABCA1 protein was expressed abundantly in exocrine glandular cells (Fig. 9C), whereas islet cells contained much less ABCA1 protein (not shown).

Elsewhere, ABCA1 protein was localized to alveolar macrophages and the lining epithelium of small bronchioles in the lung, and in the adrenal gland where ABCA1 abundance was greatest in the cortex (not shown). In the spleen, highest ABCA1 mRNA and protein expression was observed in the follicles, with enhanced staining of splenic macrophages as well as in red pulp (not shown). Cardiomyocytes and skeletal 


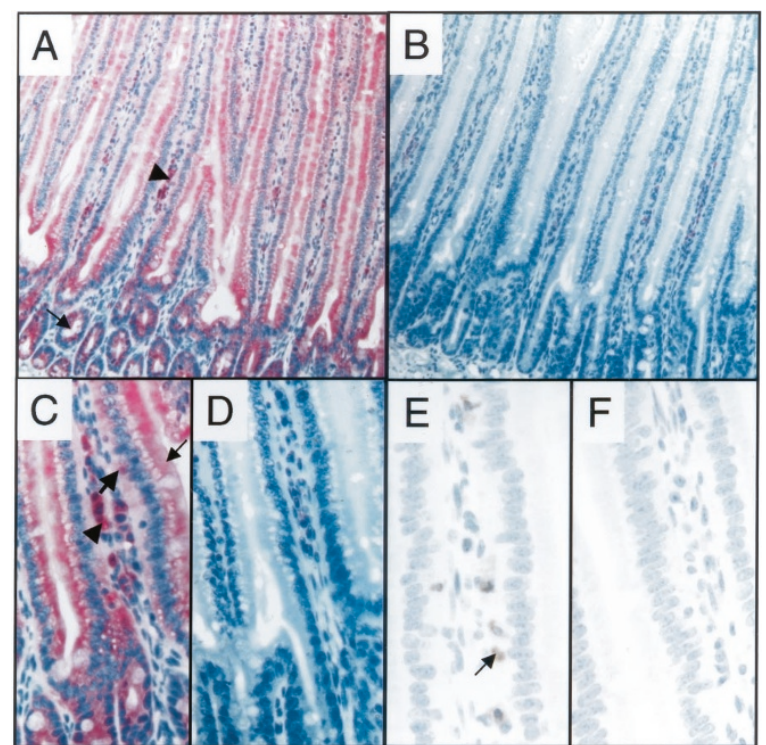

Figure 6.

Immunoreactivity of small intestine for ABCA1 protein. A, Vivid positive immunoreactivity in lining epithelial cells of intestinal villi is illustrated by Vector Red staining. Positivity is also seen in the glands of Lieberkühn (arrow) at the base of the villi. The supporting connective tissue has visibly positive interstitial cells arrayed along the vascular tributaries (arrowhead). B, Immunoreactivity is absent in the negative control. C, Higher magnification of Panel A highlights the prominence of abluminal positivity in the lining epithelium (arrow), as well as toward the basal portion of the epithelial cells (thick arrow) and in the interstitial cells of the underlying lamina propria (arrowhead). Small vascular structures running in the interstitium can also be seen. D, The negative control for Panel C. E, Immunoreactivity for macrophages (arrow) is demonstrated by monoclonal antibody staining with antibody F4/80. F, Nearly negative staining for $T$ cells is observed with an antibody to CD3. Original magnification: $A$ and $B, \times 125$; $C$ to $F, \times 330$; hematoxylin counterstain.

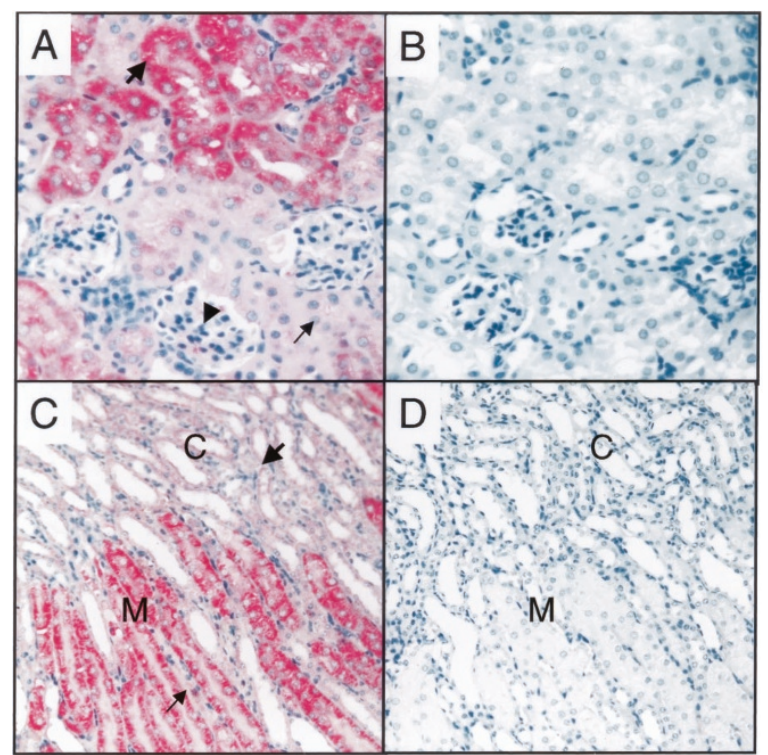

Figure 7.

Immunoreactivity of murine kidney for ABCA1 protein. A, Strong immunoreactivity (thick arrow) is observed in proximal convoluted tubular cells. In contrast, weak positivity is noted within distal convoluted tubular cells (arrow). Cells within glomeruli are generally negative. B, The negative control for Panel A. C, In the deep medulla $(M)$, one sees strong positivity in the thick ascending limb of the loop of Henle (arrow), with uniform weak but distinct positivity in collecting $(C)$ duct epithelium (thick arrow). D, The negative control for Panel C. Original magnification: $A$ and $B, \times 330 ; C$ and $D, \times 125$; hemotoxylin counterstain.

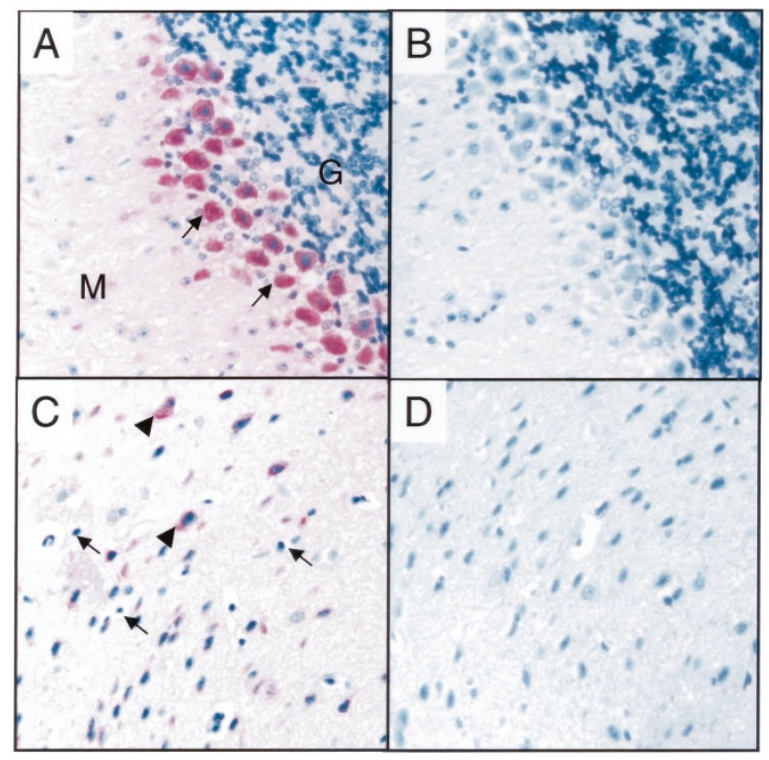

Figure 8.

Immunoreactivity of murine cerebellum and cerebrum for ABCA1 protein. A, Strong positivity is observed in Purkinje cells (arrows). Cells within the granular layer $(G)$ are generally negative. The molecular layer $(M)$ is also largely negative. B, The negative control for Panel A. C, Positivity is observed in pyramidal cells (arrowheads) of the deeper cortical layers. Neural glia are denoted (arrows). D, The negative control for Panel C. Original magnification: A to $\mathrm{D}, \times 330$; hematoxylin counterstain.

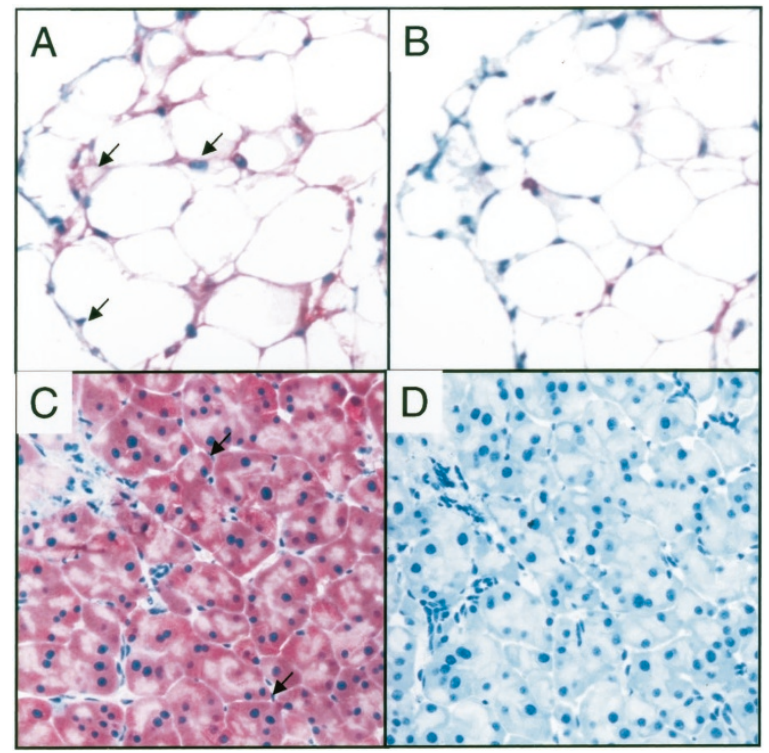

Figure 9.

Immunoreactivity of ABCA1 in adipose and pancreas tissue for ABCA1 protein. A, Immunopositivity is observed in interstitial fibroblasts between adipocytes. B, The negative control for Panel A. C, Strong immunopositivity of exocrine glandular cells of the pancreas, especially in the basal region (arrows). Interstitial microvascular cells are apparently negative. Cells within the islet were weakly positive (data not shown). D, The negative control for Panel C. Original magnification: A to D, $\times 330$; hematoxylin counterstain. 
muscle contained clear patches of cells with ABCA1 protein (not shown).

\section{ABCA1 Expression is Up-Regulated Specifically in the Liver in Response to an Atherogenic Diet}

High expression of ABCA1 in the liver strongly suggests a major role for the liver in the regulation of HDL-C levels. We have previously demonstrated that a 7-day exposure to a high-fat/high-cholesterol diet results in an increase of endogenous murine ABCA1 mRNA and protein levels in the liver as well as an increase in plasma HDL-C (Singaraja et al, 2001). To determine the specificity of dietary ABCA1 upregulation in the liver compared with other tissues, C57/BI6-CBA hybrid mice were fed a control or a high-fat/high-cholesterol diet ad libitum for 7 days, after which ABCA1 protein levels were evaluated from multiple tissues. Dietary up-regulation of ABCA1 protein was observed most markedly in the liver (3.2-fold; $n=5$; Fig. 10). These results support the existence of liver-specific regulatory pathways that increase ABCA1 protein abundance in response to diet, and suggest that diet-induced modifications in HDL-C levels may be mediated predominately through changes in liver-specific ABCA1 expression.

\section{Discussion}

The importance of $A B C A 1$ in cholesterol efflux has been recently demonstrated by the identification of ABCA1 mutations in TD and FHA families (Bodzioch et al, 1999; Brooks-Wilson et al, 1999; Marcil et al, 1999; Rust et al, 1999). The identification of ABCA1 has

A

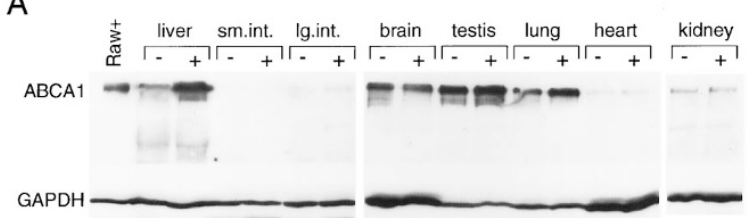

B

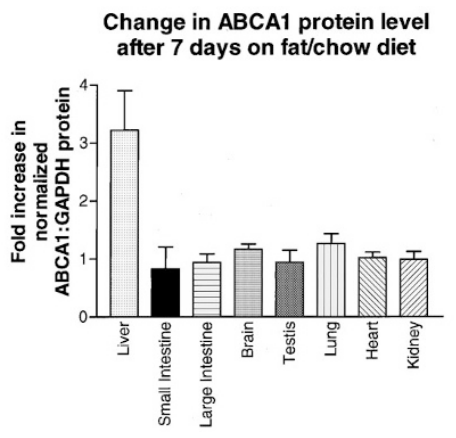

Figure 10.

Prominent up-regulation of $A B C A 1$ protein expression in liver in response to diet. Wild-type C57/BI6-CBA hybrid mice were fed a control (control) or a high-fat/high-cholesterol (fat) diet ad libitum for 7 days. A, The relative amount of $A B C A 1$ in tissues extracted from mice on the control versus the fat diet was evaluated by Western blot analysis of total cellular protein. $B, A B C A 1$ relative to GAPDH protein levels were first determined for individual tissues, and then expressed as the ratio of $A B C A 1$ in fat versus chow-fed animals following densitometric quantitation. launched intensive investigations into the possibility of providing protection from atherosclerosis by increasing $A B C A 1$ expression and thereby raise plasma HDL-C levels. Because it is desirable to target potential therapeutics to the cells directly involved in HDL-C regulation, a key initial step toward this goal is to understand the normal distribution patterns of ABCA1 expression. Furthermore, it is essential that expression of $A B C A 1$ be investigated at the protein level because protein levels cannot accurately be predicted from mRNA expression patterns (Gygi et al, 1999).

Here we provide the first report of ABCA1 protein distribution in the normal mouse. In whole tissues, high levels of ABCA1 protein were found in liver, testis, and adrenal gland. These results suggest that hepatic ABCA1 is likely to play a key role in the regulation of HDL-C levels, a hypothesis that is supported by the observation of increased ABCA1 protein expression specifically in the liver of fat-fed mice. Interestingly, liver-specific transcripts encoding an alternatively spliced form of ABCA1 have previously been reported (Cavelier et al, 2001; Pullinger et al, 2000; Singaraja et al, 2001), and a specific ABCA1 mRNA species has also been identified in hepatocytes (Costet et al, 2000). Whether these transcripts are specifically upregulated in response to an atherogenic diet is not yet known. Providing further support for this hypothesis is the observation that $A B C A 1$ mRNA is widely expressed in baboon, including in hepatocytes and Kupffer cells (Lawn et al, 2001), although ABCA1 protein levels and the responsiveness of $A B C A 1$ expression to diet in primate models has yet to be determined.

ABCA1 protein was also highly expressed in the lining epithelia of the entire gut. In the intestine, the majority of ABCA1 protein was located in the cytoplasm of epithelial cells. In the normal baboon, ABCA1 mRNA was found predominately in the intestinal lamina propria and appeared to be absent from epithelial cells, a point noted by the authors as being inconsistent with the proposed function of ABCA1 in absorption of dietary cholesterol (Lawn et al, 2001; McNeish et al, 2000). However, because ABCA1 protein levels were not examined in the baboon study, it is possible that ABCA1 protein exists in the baboon gut epithelia although ABCA1 mRNA levels were below the detection limit. In the murine intestinal villus, we observed a gradient of ABCA1 mRNA expression that was most abundant in crypts and diminished to virtually undetectable levels at the villus tips. Nonetheless, ABCA1 protein was expressed strongly even in epithelial cells at the tips of villi, suggesting that ABCA1 protein can clearly be present in gut epithelial cells in the absence of detectable mRNA levels.

The abundance of $A B C A 1$ protein in the proximal convoluted tubule of the kidney suggests that ABCA1 may participate in the regulation of membrane content of tubular cells, where sphingomyelin and cholesterol have been shown to control the accessibility and/or activity of apical cotransport systems (Vrtovsnik et al, 1992). ABCA1 may also participate in the regulation of the cholesterol content of proximal tubule cells after ischemic or toxic injury, where the cholesterol content 
is dramatically increased to stabilize the plasma membrane (Zager, 2000; Zager et al, 1999). Whether ABCA1-deficient mice are at increased risk for renal damage after ischemia has not been tested. However, ABCA1-deficient mice have been reported to develop membranoproliferative glomerulonephritis by 6 months of age due to immunoglobulin and C3 complement deposition (Christiansen-Weber et al, 2000). Membranoproliferative glomerulonephritis type II, which is associated with intramembranous dense deposits, is also associated with partial lipodystrophy, a rare autosomal recessive disorder characterized by loss of adipose tissue in the face and upper body (Demetriou et al, 1998; Levy et al, 1998; Pollock et al, 1986; Power and Simpson, 1990). Additionally, lipid-rich diets have been shown to increase the proliferation of mesangial cells in the kidney and contribute to the development of glomerulosclerosis by the accumulation of lipid deposits and foam cells in mesangial areas (Song et al, 2000). The presence of ABCA1 in murine and baboon (Lawn et al, 2001) tubular epithelia and glomerula is consistent with the hypothesis that defects in cholesterol efflux in the kidney may contribute to glomerulonephritis. Of note is that ABCA1 protein levels in the kidney were unaffected by dietary cholesterol content.

We also observed ABCA1 mRNA and protein in murine brain in several neuronal populations, including Purkinje, cortical, and striatal neurons (Singaraja et al, 2001). Although a role for ABCA1 in the brain is not currently understood, our results are consistent with the possibility that ABCA1 in the brain may participate in lipoprotein metabolism in the central nervous system, which is known to be regulated largely independently of that in plasma (Danik et al, 1999; Ladu et al, 2000). ABCA1 has been shown to efflux cholesterol to apo-E (Bortnick et al, 2000; Remaley et al, 2001), which is the predominant apolipoprotein in the central nervous system (Danik et al, 1999).

High levels of ABCA1 protein identified in the testes and adrenal gland suggest that $A B C A 1$ may play a prominent role in delivering cholesterol for steroid biosynthesis, which is supported by the observation of altered steroid hormone levels and vacuolated testes in ABCA1-deficient mice (Christiansen-Weber et al, 2000).

Overall, our results are largely consistent with a recent survey of $A B C A 1$ mRNA expression in the normal baboon, where in situ hybridization was used to demonstrate ABCA1 mRNA in resident inflammatory cells and lymphocytes in several tissues, as well as in hepatocytes, collecting tubules of the kidney, and Leydig cells in the testes (Lawn et al, 2001). ABCA1 was also identified in glia and in cerebellar granule neurons (Lawn et al, 2001), suggesting that $\mathrm{ABCA} 1$ has a yet to be characterized role in the central nervous system. Although these studies have reported expression patterns of ABCA1 mRNA (Kaminski et al, 2000; Langmann et al, 1999), the distribution of ABCA1 protein has not previously been addressed.

Our results also demonstrate that $A B C A 1$ mRNA levels were poor indicators of ABCA1 protein abundance in many tissues. These observations suggest that expression of $A B C A 1$ may in part be regulated post-transcriptional mechanisms in selected cells or tissues and that these regulatory mechanisms may require consideration in the development of potential therapies designed to increase ABCA1 expression. Furthermore, these results suggest that ABCA1 protein abundance is a more important primary outcome measure than mRNA levels.

Our results support the existence of liver-specific pathways that regulate $A B C A 1$ expression in response to dietary fat. Transcription of $A B C A 1$ has been shown to be induced by cAMP as well as by LXR-RXR pathways in macrophages (Abe-Dohmae et al, 2000; Bortnick et al, 2000; Costet et al, 2000; Oram et al, 2000; Pullinger et al, 2000; Repa et al, 2000; Schwartz et al, 2000). These observations suggest that alteration of ABCA1 expression in macrophages with compounds that affect ABCA1 expression may help to reduce the transition to foam cells and decrease the number or rate of progression of atherosclerotic plaques. However, up-regulation of $A B C A 1$ expression is not observed in many cell types (Bortnick et al, 2000). Therefore, potential therapeutic approaches designed to increase ABCA1 expression must also consider all possible functions of ABCA1, many of which still remain to be determined. Initial clues, however, are their cellular sites of expression reported in this manuscript.

\section{Materials and Methods}

\section{Semi-Quantitative RT-PCR}

All animal studies were performed according to protocols approved by the Ethics Review Committee of the University of British Columbia. Tissues from four adult FVB mice were snap frozen in liquid nitrogen and stored at $-70^{\circ} \mathrm{C}$. RNA extraction was performed using a RNeasy Kit (Qiagen, Mississauga, Ontario) according to the manufacturer's instructions. RNA was then either stored at $-70^{\circ} \mathrm{C}$ or quantified immediately using the Ultrospec 3000 UV/Visible Spectrophotometer (Pharmacia Biotech, Piscataway, New Jersey) before two-step RT-PCR protocol using $4 \mu \mathrm{g}$ of total RNA. RNA was treated with DNAse I (Roche, Laval, Québec), reverse transcribed (MMLV Reverse Transcriptase; Canadian Life Technologies, Burlington, Ontario), and amplified for ABCA1 or 18s rRNA using Qiagen PCR enzymes and reagents according to the following conditions: 15 minutes $95^{\circ} \mathrm{C}$, then 40 cycles of 1 minute $95^{\circ} \mathrm{C}, 1$ minute $55^{\circ} \mathrm{C}$, and 1 minute $72^{\circ} \mathrm{C}$, and then a final annealing step at $72^{\circ} \mathrm{C}$ for 7 minutes. All thermocycling was performed on a Perkin Elmer Gene Amp PCR System 9700 (Ville St-Laurent, Québec). PCR amplification was performed using either mABC1 primers (forward: 5'CAAACATGTRCAGCTGTTACTGGA-3' and reverse: 5'-TAGCCTTGCAAAAATACCTTCTG-3') (Langmann et al, 1999) or the Universal 18s rRNA primer:competimer system (Ambion, Austin, Texas). PCR products were separated on $2 \%$ agarose gels containing 
ethidium bromide. Densitometric quantitation of the intensity of the $18 \mathrm{~s}$ rRNA and ABCA1 products was determined using the Eagle Eye II Still Video System (Stratagene, Cedar Creek, Texas). The relative abundance of $A B C A 1$ was expressed as the ratio of ABCA1: 18s rRNA product.

\section{Antibody Development and Verification}

A synthetic peptide corresponding to amino acids (aa) 2236-2259 of the human ABCA1 sequence (HKNQTVVDVAVLTSLQDEKVKES) was used to generate a polyclonal ABCA1-specific antibody, and a monoclonal antibody was raised against a GST-fusion protein containing the C-terminal domain of ABCA1 (aa 18742261). ABCA1 constructs used for antibody validation were each expressed from the cytomegalovirus promoter in pcDNA3 (InVitrogen, Burlington, Ontario). One construct included the full-length human ABCA1 cDNA encoding 2261 aa, and $\mathrm{N}$-terminal and C-terminal expression constructs were prepared from full-length ABCA1 cDNA and encoded the first 638 aa or the final 387 aa, respectively. All constructs were verified by sequence analysis. In vitro translation was performed using the TNT Quick Coupled Rabbit Reticulocyte system (Promega, Madison, Wisconsin) according to the manufacturer's recommendations.

\section{Cell Culture}

Human embryonic kidney 293T cells were cultured in DMEM (Canadian Life Technologies) with 10\% FCS, $50 \mathrm{U} / \mathrm{ml}$ penicillin-streptomycin, and $2 \mathrm{~mm}$ L-glutamine and transiently transfected using calcium phosphate. Cells were harvested 24 hours after transfection by gentle scraping. RAW murine macrophages and human fibroblasts were maintained in DMEM (Canadian Life Technologies) with 10\% FCS, $50 \mathrm{U} / \mathrm{ml}$ penicillinstreptomycin, $2 \mathrm{~mm}$ L-glutamine, $1 \times$ nonessential amino acids (Canadian Life Technologies), and 10 ng/ml EGF (Sigma, Oakville, Ontario). To stimulate ABCA1 expression, growth media was replaced with media containing delipidated serum (Sigma) and either ethanol (control) or $10 \mu \mathrm{M}$ 9-cis retinoic acid (RA) (10 $\mathrm{mg} / \mathrm{ml}$ stock in ethanol; Sigma) and $4 \mu \mathrm{g} / \mathrm{ml} 22-\mathrm{R}-\mathrm{OH}-$ cholesterol $(4 \mathrm{mg} / \mathrm{ml}$ stock in ethanol; Steraloids, Newport, Rhode Island) for up to 24 hours.

\section{Dietary Regulation of ABCA1 Expression}

Dietary regulation of ABCA1 expression was determined as described previously (Singaraja et al, 2001). Wild-type C57/BI6-CBA hybrid mice were maintained on a control diet or a high-fat/high-cholesterol diet for 7 days. The diets and water were provided ad libitum. Diets were purchased from Harlan Teklad with the high-fat/high-cholesterol diet (TD 90221) containing $15.75 \%$ fat, $1.25 \%$ cholesterol, and $0.5 \%$ sodium cholate; these diets have been shown to result in up-regulation of ABCA1 mRNA levels in mouse liver, assessed at 7 days after feeding (Hedrick et al, 2000). The control diet contained $0.5 \%$ sodium cholate (TD
99057). Tissues were harvested from three to five independent animals.

\section{Protein Isolation and Western Blotting}

Cell pellets were lysed by gentle tritration in $20 \mathrm{~mm}$ Hepes, $5 \mathrm{~mm} \mathrm{KCl}, 5 \mathrm{~mm} \mathrm{MgCl}, 0.5 \%$ (v/v) Triton $\mathrm{X}-100$, and complete protease inhibitor (Roche). Cell debris was removed by centrifugation at $5,000 \mathrm{rpm}$ for 4 minutes at $4^{\circ} \mathrm{C}$. Tissues were homogenized in an ice-cold buffer containing $20 \mathrm{~mm}$ Hepes, $5 \mathrm{~mm} \mathrm{KCl}, 5$ $\mathrm{mM} \mathrm{MgCl}_{2}, 0.5 \%(\mathrm{v} / \mathrm{v})$ Triton X-100, and complete protease inhibitor (Roche). Homogenates were sonicated once for 10 seconds followed by centrifugation at $12,000 \mathrm{rpm}$ for 5 minutes at $4^{\circ} \mathrm{C}$. The protein concentration in tissue or cellular supernatants were determined by the Lowry assay. Equal amounts of protein (typically $80 \mu \mathrm{g}$ ) were separated on $7.5 \%$ SDS-PAGE gels and electrophoretically transferred to PVDF membrane (Millipore, Toronto, Ontario). Membranes were probed with either ABCA1 antibody or a monoclonal anti-GAPDH antibody (Chemicon, Mississauga, Ontario) as a control for equal loading. Immunoreactivity was detected by ECL (Amersham, Baie D'Urfé, Québec). Protein abundance was calculated by densitometry using $\mathrm{NIH} \mathrm{Image} \mathrm{version} 6.0$ software and normalized to GAPDH levels in each tissue.

\section{Tissue Processing and Hematoxylin and Eosin Staining}

Murine tissues (FVB) were composited and fixed in 10\% neutral buffered formalin (Fisher-Scientific, Edmonton, Alberta). Overnight tissue processing was performed using a Shandon Pathcentre (Pittsburgh, Pennsylvania) tissue processor. Tissue samples were then embedded in paraffin and contiguous $3-\mu \mathrm{m}$ microtome sections were used for detection of $A B C A 1$ mRNA or protein. Staining was automated using the Tissue-Tek DRS2000 stainer (Torrance, California).

\section{In Situ Hybridization}

In situ hybridization was performed as previously described (Anderson et al, 1996). Briefly, tissues on slides were permeabilized, acetylated, and dehydrated in graded alcohols, dried, and hybridized using digoxigenin-labeled $A B C A 1$ anti-sense riboprobes or CVB3 control riboprobes at $55^{\circ} \mathrm{C}$ overnight. A murinespecific ABCA1 anti-sense riboprobe was prepared by in vitro transcription (Promega) from a 605-bp cDNA fragment spanning nt positions 1 through 605 of the murine ABCA1 sequence (GenBank accession X75926). The CVB3 control riboprobe was included as a negative control and has been previously described (Komminoth, 1996). After hybridization, slides were washed for 30 minutes in formamide, 2x SSC (1:1), 20 minutes in 2x SSC, and twice for 20 minutes in $0.2 x$ SSC. Detection of signal used sheep anti-digoxygenin polyclonal antibody conjugated to alkaline phosphatase (Boehringer-Mannheim, Laval, Québec) and VECTOR Red (Vector Laboratories, Burlington, Ontario) as a substrate. Darkfield images were viewed through a TE-FM Epi-fluorescence filter (excitation 
510-560 nm). Images were captured using the SPOT camera hardware attachment and corresponding software (Diagnostic Instruments, Sterling Heights, Michigan).

\section{ABCA1 Immunohistochemistry}

Prepared slides were deparaffinized in graded alcohols reaching $95 \%$. After a 5 -minute incubation in $1.5 \% \mathrm{H}_{2} \mathrm{O}_{2}$ in methanol, slides were washed in water and rinsed in Tris-buffered saline (TBS) (pH 7.6). Slides were placed in sodium citrate buffer ( $\mathrm{pH} 6)$, steamed for 30 minutes, washed in TBS, and blocked for 30 minutes in 1:20 normal goat serum (Vector) in TBS. For the polyclonal antibody, ABCA1 was detected with an overnight incubation of primary antibody and then washing in TBS and incubation with goat anti-rabbit antibody (Vector) for 40 minutes. Peroxidase conjugated streptavidin (DAKO) was then applied for 30 minutes with washes in TBS both before and after the incubation. DAB chromagen solution (Sigma) was added for the final detection step. After a water wash, slides were counterstained with hematoxylin, washed, dehydrated, and mounted. Images were recorded using the SPOT camera system with a Nikon upright microscope. Controls included omission of primary antibody (no staining observed), and inclusion of an unrelated polyclonal primary antibody (a different pattern of expression observed). Detection of ABCA1 using the monoclonal antibody staining was performed using the same procedures except for blocking in normal horse serum (Vector) and eliminating the hydrogen peroxidase block. Cultured hybridoma cell medium was used as a primary antibody. Vector Red was used as a chromagen against a hematoxylin counterstain.

\section{CD3 and F4/80 Immunohistochemistry}

$T$ cells were detected by using the rat anti-human CD3 (Serotec, Hornby, Ontario) and then biotin-conjugated anti-rat secondary antibody (Ventana, Tucson, Arizona) and the Basic DAB Detection Kit (Ventana) using the automated Ventana detection system. Murine macrophages were detected using the rat antihuman F4/80 antibody (Serotec). Slides were initially digested with $0.1 \%$ protease 1 (Ventana) diluted in TBS for 15 minutes at $37^{\circ} \mathrm{C}$, then blocked in normal goat serum for 30 minutes and incubated with the F4/80 antibody for 2 hours. Slides were washed in TBS, incubated with goat anti-rat antibody (Ventana) for 45 minutes, and washed. Chromogen detection with DAB (Sigma) was performed as above.

\section{Acknowledgements}

The authors wish to thank Dr. Joan Keiser, Dr. Sherrie Tafuri, Dr. Ming Wang, Dr. Mark Gray-Keller, and Dr. Alan Attie for their generous intellectual contributions to this work, and members of our respective groups for their constructive suggestions. The authors would also like to thank Ms. Liwina Pang, Ms. Linda Hughes, Mr. Albert Lee, Ms. Amrit Mahil, and Ms.
Zong Shu Luo for their excellent technical advice and assistance.

\section{References}

Abe-Dohmae S, Suzuki S, Wada Y, Aburatani H, Vance DE, and Yokoyama $S$ (2000). Characterization of apolipoproteinmediated HDL generation induced by cAMP in a murine macrophage cell line. Biochemistry 39:11092-11099.

Anderson DR, Wilson JE, Carthy CM, Yang D, Kandolf R, and McManus BM (1996). Direct interactions of Coxsackivirus B3 with immune cells in the splenic compartment of mice susceptible or resistant to myocarditis. J Virol 70:4632-4645.

Bodzioch M, Orsó E, Klucken J, Langmann T, Böttcher A, Diederich W, Drobnik W, Barlage S, Büchler C, PorschÖzcürümez M, Kaminski WE, Hahmann HW, Oette K, Rothe G, Aslanidis C, Lackner KJ, and Schmitz G (1999). The gene encoding ATP-binding cassette transporter 1 is mutated in Tangier Disease. Nat Genet 22:347-351.

Bortnick AE, Rothblat GH, Stoudt G, Hoppe KL, Royer LJ, McNeish J, and Francone OL (2000). The correlation of ATP-binding cassette $1 \mathrm{mRNA}$ levels with cholesterol efflux from various cell lines. J Biol Chem 275:28634-28640.

Broccardo C, Luciani M, and Chimini G (1999). The ABCA subclass of mammalian transporters. Biochim Biophys Acta 1461:395-404.

Brooks-Wilson A, Marcil M, Clee SM, Zhang L, Roomp K, van Dam M, Yu L, Brewer C, Collins JA, Molhuizen HOF, Loubser O, Ouellette BFF, Fichter K, Ashbourne Excoffon KJD, Sensen CW, Scherer S, Mott S, Denis M, Martindale D, Frohlich J, Morgan K, Koop B, Pimstone SN, Kastelein JJP, Genest J Jr, and Hayden MR (1999). Mutations in ABC1 in Tangier disease and familial high-density lipoprotein deficiency. Nat Genet 22:336-345.

Cavelier LB, Qiu Y, Bielicki JK, Afzal V, Cheng J-F, and Rubin EM (2001). Regulation and activity of the human ABCA1 gene in transgenic mice. J Biol Chem 276:18046-18051.

Christiansen-Weber TA, Voland JR, Wu Y, Ngo K, Roland BL, Nguyen S, Peterson PA, and Fung-Leung WP (2000). Functional loss of ABCA1 in mice causes severe placental malformation, aberrant lipid distribution, and kidney glomerulonephritis as well as high-density lipoprotein cholesterol deficiency. Am J Pathol 157:1017-1029.

Costet P, Luo Y, Wang N, and Tall AR (2000). Steroldependent transactivation of the ABC1 promoter by the liver $X$ receptor/retinoid X receptor. J Biol Chem 275:2824028245.

Danik M, Champagne D, Petit-Turcotte C, Beffert U, and Poirer J (1999). Brain lipoprotein metabolism and its relation to neurodegenerative disease. Crit Rev Neurobiol 13:357407.

Demetriou K, Kallikas I, Zouvan I, Kyriacou K, and Pierides A (1998). The pregnant patient with partial lipodystrophy developing acute renal failure - onset of de novo membranoproliferative glomerulonephritis. Nephrol Dial Transplant 13: 2121-2124.

Gygi SP, Rochon Y, Franza BR, and Aebersold R (1999). Correlation between protein and mRNA abundance in yeast. Mol Cell Biochem 19:1720-1730.

Hedrick CC, Hassan K, Hough GP, Yoo JH, Simzar S, Quinto CR, Kim SM, Dooley A, Langi S, Hama SY, Navab M, Witztum $\mathrm{JL}$, and Fogelman AM (2000). Short-term feeding of an 
atherogenic diet to mice results in reduction of $\mathrm{HDL}$ and paraoxonase that may be mediated by an immune mechanism. Arterioscler Thromb Vasc Biol 20:1946-1952.

Kaminski WE, Orsó E, Diederich W, Klucken J, Drobnik W, and Schmitz G (2000). Identification of a novel human sterolsensitive ATP-binding cassette transporter (ABCA7). Biochem Biophys Res Commun 273:532-538.

Klein I, Sarkadi B, and Váradi A (1999). An inventory of the human ABC proteins. Biochim Biophys Acta 1461:237-262.

Komminoth P (1996). Detection of mRNA in tissue sections using DIG-labeled RNA and oligonucleotide probes. In: Nonradioactive in situ hybridization manual, 2 nd ed. Mannheim: Boehringer Mannheim GmbH, 126-135.

Ladu MJ, Reardon C, Van Eldik L, Fagan AM, Bu G, Holtzmann D, and Getz GS (2000). Lipoproteins in the central nervous system. Ann NY Acad Sci 903:167-175.

Langmann T, Klucken J, Reil M, Liebisch G, Luciani M-F, Chimini G, Kaminski WE, and Schmitz G (1999). Molecular cloning of the human ATP-binding cassette transporter 1 (hABC1): Evidence for sterol-dependent regulation in macrophages. Biochem Biophys Res Commun 257:29-33.

Lawn RM, Wade DP, Couse TL, and Wilcox JN (2001). Localization of human ATP-Binding cassette transporter 1 (ABC1) in normal and atherosclerotic tissues. Arterioscler Thromb Vasc Biol 21:378-385.

Levy Y, George J, Yona E, and Shoenfeld Y (1998). Partial lipodystrophy, mesangiocapillary glomerulonephritis, and complement dysregulation. An autoimmune phenomenon. Immunol Res 18:55-60.

Marcil M, Brooks-Wilson A, Clee SM, Roomp K, Zhang L, Yu L, Collins JA, van Dam M, Molhuizen HOF, Loubser O, Ouellette BFF, Sensen CW, Fichter K, Mott S, Denis M, Boucher B, Pimstone S, Genest J Jr, Kastelein JJP, and Hayden MR (1999). Mutations in the ABC1 gene in familial HDL deficiency with defective cholesterol efflux. Lancet 354:1341-1346.

McNeish J, Aiello RJ, Guyot D, Turi T, Gabel C, Aldinger C, Hoppe KL, Roach ML, Royer LJ, de Wet J, Broccardo C, Chimini G, and Francone OL (2000). High density lipoprotein deficiency and foam cell accumulation in mice with targeted disruption of ATP-binding cassette transporter-1. Proc Natl Acad Sci 97:4245-4250.

Oram JF, Lawn RM, Garvin MR, and Wade DP (2000). ABCA1 is the CAMP-inducible apolipoprotein receptor that mediates cholesterol secretion from macrophages. J Biol Chem 275: 34508-34511.

Oram JF and Vaughan AM (2000). ABCA1-mediated transport of cellular cholesterol and phosopholipids to HDL apoplipoproteins. Curr Opin Lipidol 11:253-260.

Pollock J, Wood B, and Kelly JP (1986). Membranoproliferative glomerulonephritis, type II and partial lipodystrophy in an adult. Am J Kidney Dis 8:274-276.
Power DA and Simpson JG (1990). Familial incidence of C3 nephritic factor, partial lipodystrophy and membranoproliferative glomerulonephritis. Q J Med 75:387-398.

Pullinger CR, Hakamata H, Duchateau PN, Eng C, Aouizerat $\mathrm{BE}$, Cho MH, Fielding CJ, and Kane JP (2000). Analysis of hABC1 Gene 5' End: Additional peptide sequence, promoter region, and four polymorphisms. Biochem Biophys Res Commun 271:451-455.

Remaley AT, Stonik JA, Demosky SJ, Neufeld EB, Bocharov AV, Vishnyakova TG, Eggerman TL, Patterson AP, Duverger NJ, Santamarina-Fojo SS, and Brewer HB (2001). Apoplipoprotein specificity for lipid efflux by the human $A B C A 1$ transporter. Biochem Biophys Res Commun 280:818-823.

Repa JJ, Turley SD, Lobaccaro JA, Medina J, Li L, Lustig K, Shan B, Heyman RA, Dietschy JM, and Mangelsdorf DJ (2000). Regulation of absorption and ABC1-mediated efflux of cholesterol by RXR heterodimers. Science 289:15241529.

Rust S, Rosier M, Funke H, Amoura Z, Piette J-C, Deleuze J-F, Brewer HB Jr, Duverger N, Denèfle P, and Assmann G (1999). Tangier Disease is caused by mutations in the gene encoding ATP-binding cassette transporter 1. Nat Genet 22:352-355.

Schmitz G, Kaminski WE, and Orsó E (2000). ABC transporters in cellular lipid trafficking. Curr Opin Lipidol 11:493-501.

Schwartz K, Lawn RM, and Wade DP (2000). ABC1 gene expression and ApoA-1-mediated cholesterol efflux are regulated by LXR. Biochem Biophys Res Commun 274:794802.

Singaraja RR, Bocher V, James ER, Clee SM, Zhang LH, Leavitt BR, Tan B, Brooks-Wilson A, Kwok A, Bissada N, Yang YZ, Liu G, Tafuri SR, Fievet C, Wellington CL, Staels B, Hayden MR (2001). Human ABCA1 BAC transgenic mice show increased high density lipoprotein cholesterol and ApoAl-dependent efflux stimulated by an internal promoter containing liver $X$ receptor response elements in intron 1 . J Biol Chem 276:33969-33979.

Song H, Li X, Zhu C, and Wei M (2000). Glomerulosclerosis in adriamycin-induced nephrosis is accelerated by a lipid-rich diet. Pediatr Nephrol 15:196-200.

Vrtovsnik F, Yandouzi EH, Le Grimellec C, and Friedlander G (1992). Sphingomyelin and cholesterol modulate sodium coupled uptake in proximal tubular cells. Kidney Int 41:983991.

Zager RA (2000). Plasma membrane cholesterol: A critical determinant of cellular energetics and tubular resistance to attack. Kidney Int 58:193-205.

Zager RA, Burkhart KM, Johnson AC, and Sacks BM (1999). Increased proximal tubular cholesterol content: Implications for cell injury and "acquired cytoresistance." Kidney Int 56:1788-1797. 